To appear in the Proceedings of

the 19th International Conference on Software Technology and Theoretical Computer Science

\title{
Analysis of Quantum Functions
}

\author{
(Preliminary Version) \\ Tomoyuki Yamakami \\ Department of Computer Science, Princeton University \\ Princeton, New Jersey 08544
}

\begin{abstract}
Quantum functions are functions that are defined in terms of quantum mechanical computation. Besides quantum computable functions, we study quantum probability functions, which compute the acceptance probability of quantum computation. We also investigate quantum gap functions, which compute the gap between acceptance and rejection probabilities of quantum computation.
\end{abstract}

\section{Introduction}

A paradigm of quantum mechanical computers was first proposed in early 1980's $[2,10]$ to gain more computational power over classical computers. Recent discoveries of fast quantum algorithms for a variety of problems have raised much enthusiasm among computer scientists as well as physicists. These discoveries have supplied general and useful tools in programming quantum algorithms.

We use in this paper a multi-tape quantum Turing machine (abbreviated a QTM) $[6,4]$ as a mathematical model of a quantum computer. A well-formed QTM can be identified with a unitary operator, so-called a time-evolution operator, that performs in the infinite dimensional space of superpositions, which are linear combinations of configurations of the QTM.

The main theme of this paper is a study of polynomial time-bounded quantum functions. A quantum computable function, a typical quantum function, computes an output of a QTM with high probability. Let FQP and FBQP denote the collections of functions computed by polynomial-time, well-formed QTMs, respectively, with certainty and with probability at least 2/3. A quantum probability function, on the contrary, computes the acceptance probability of a QTM. For notational convenience, \#QP denotes the collection of such quantum functions particularly witnessed by polynomial-time, well-formed QTMs. Another important quantum function is the one that computes the gap between the acceptance and rejection probabilities of a QTM. We call such functions quantum gap functions and use the notation GapQP to denote the collection of polynomial-time quantum gap functions.

In this paper, we explore the characteristic feature of these FQP-, FBQP-, \#QP-, and GapQP-functions and study the close connection between GapQPfunctions and classical GapP-functions. One of the most striking features of

\footnotetext{
* This work was partly supported by NSERC Fellowship and DIMACS Fellowship.
} 
quantum gap functions is that if $f \in \mathbf{G a p Q P}$ then $f^{2} \in \# \mathbf{Q P}$, where $f^{2}(x)=$ $(f(x))^{2}$.

We also study relativized quantum functions that can access oracles to help their computation. We exhibit an oracle $A$ showing that $\mathbf{F Q P}{ }^{A}$ is more powerful than $\# \mathbf{P}^{A}$ and also show the existence of another oracle $A$ such that $\# \mathbf{Q P} \mathbf{P}^{A}$ is not included in non-adaptive version of $\# \mathbf{Q P} \mathbf{P}^{A}$.

\section{Basic Notions and Notation}

We use the standard notions and notation that are found elsewhere. In this section, we explain only what needs special attention.

Let $\mathbb{A}$ denote the set of complex algebraic numbers and let $\tilde{\mathbb{C}}$ denote the set of complex numbers whose real and imaginary parts can be approximated to within $2^{-n}$ in time polynomial in $n$. We freely identify any natural number with its binary representation. When we discuss integers, we also identify each integer with its binary representation following a sign bit that indicates the (positive or negative) $\operatorname{sign}^{\star \star}$ of the integer. Moreover, a rational number is also identified as a pair of integers, which are further identified as binary integers.

Let $\mathbb{N}^{\Sigma^{*}}$ be the set of all functions that map $\Sigma^{*}$ to $\mathbb{N}$. Similarly, we define $\{0,1\}^{\Sigma^{*}}, \mathbb{N}^{\mathbb{N}}$, etc. We identify a set $S$ with its characteristic function, which is defined as $S(x)=1$ if $x \in S$ and 0 otherwise. In this paper, a polynomial with $k$ variables means an element in $\mathbb{N}\left[x_{1}, x_{2}, \ldots, x_{k}\right]$.

Assumed is the reader's familiarity with central complexity classes, such as $\mathbf{P}, \mathbf{N P}, \mathbf{B P P}, \mathbf{P P}$, and PSPACE and function classes, such as $\mathbf{F P}, \# \mathbf{P}$ [13], and $\mathbf{G a p P}[8]$. Note that $\mathbf{F P} \subseteq \# \mathbf{P} \subseteq \mathbf{G a p P}$.

The notion of a quantum Turing machine - abbreviated a QTM-was originally introduced in [6] and developed in [4]. For convenience, we use in this paper a slightly more general definition of QTMs defined in [14]; a $k$-tape QTM $M$ is a quintuple $\left(Q,\left\{q_{0}\right\}, Q_{f}, \Sigma_{1} \times \Sigma_{2} \times \cdots \times \Sigma_{k}, \delta\right)$, where each $\Sigma_{i}$ is a finite alphabet with a distinguished blank symbol \#, $Q$ is a finite set of states including an initial state $q_{0}$ and a set $Q_{f}$ of final states, and $\delta$ is a multi-valued quantum transition function from $Q \times \Sigma_{1} \times \Sigma_{2} \times \cdots \times \Sigma_{k}$ to $\mathbb{C}^{Q \times \Sigma_{1} \times \Sigma_{2} \times \cdots \times \Sigma_{k} \times\{L, R, N\}^{k}}$. The QTM has $k$ two-way infinite tapes of cells indexed by $\mathbb{Z}$ and its read/write heads that move along the tapes either to the left or to the right, or the heads stay still. In particular, the last tape of $M$ is used to write an output. It is known in $[4,16$, $14]$ that our model is polynomially "equivalent" to more restrictive model as in [4], which is called conservative in [14].

Let $M$ be a QTM. The running time of $M$ on input $x$ is defined to be the minimal number $T$, if any, such that, at time $T$, every computation path of $M$ on $x$ reach a certain final configuration. We say that $M$ on input $x$ halts in time $T$ if its running time is defined and equals $T$. We call $M$ a polynomial-time $Q T M$ if there exists a polynomial $p$ such that, for every $x, M$ on input $x$ halts in time $p(|x|)$. A QTM is well-formed if its time-evolution operator preserves the

\footnotetext{
** For example, we set 1 for a positive integer and 0 for a negative integer.
} 
$L_{2}$-norm. For any $x$, the notation $\rho_{M}(x)$ denotes the acceptance probability of $M$ on $x$ : that is, the sum of every squared magnitude of the amplitude, in the final superposition of $M$, of any configuration whose output tape constitutes a symbol "1" (called an accepting configuration). For a nonempty subset $K$ of $\mathbb{C}$, we say that $M$ has $K$-amplitudes if the entries of its time-evolution matrix are all drawn from $K$. For more notions and terminology (e.g., stationary, synchronous, normal form), the reader refers to $[4,14]$.

We also use an oracle QTM that is equipped with an extra tape, called a query tape and two distinguished states, a pre-query state $q_{p}$ and a post-query state $q_{a}$. The oracle QTM invokes an oracle query by entering state $q_{p}$. In a single step, the content $|y \circ b\rangle$ of the query tape, where $b \in\{0,1\}$ and "o" denotes concatenation, is changed into $|y \circ(b \oplus A(y))\rangle$ and the machine enters state $q_{a}$.

For a superposition $|\phi\rangle$, the notation $M(|\phi\rangle)$ denotes the final superposition of $M$ that starts with $|\phi\rangle$ as an initial superposition.

There are two useful unitary transforms used in this paper. The phase shift $P$ maps $|0\rangle$ to $-|0\rangle$ and $|1\rangle$ to $|1\rangle$. The Hadamard transform $H$ changes $|0\rangle$ into $\frac{1}{\sqrt{2}}(|0\rangle+|1\rangle)$ and $|1\rangle$ into $\frac{1}{\sqrt{2}}(|0\rangle-|1\rangle)$.

Throughout this paper, $K$ denotes an arbitrary subset of $\mathbb{C}$ that includes $\{0, \pm 1\}$. All quantum function classes discussed in this paper depend on the choice of $K$-amplitudes. We find it convenient to drop script $K$ when $K=\tilde{\mathbb{C}}$.

\section{Various Quantum Functions}

In this section, we formally define a variety of quantum functions and discuss their fundamental properties. Recall that $\{0, \pm 1\} \subseteq K \subseteq \mathbb{C}$

3.1 Exact Quantum Computable Functions. We begin with quantum functions whose values are the direct outputs, with certainty, of polynomial-time, well-formed QTMs. We call them exact quantum polynomial-time computable in a similar fashion to polynomial-time computable functions.

Definition 1. Let $\mathbf{F Q P} \mathbf{P}_{K}$ be the set of $K$-amplitude exact quantum polynomialtime computable functions; that is, there exists a polynomial-time, well-formed QTM with $K$-amplitudes such that, on every input $x, M$ outputs $f(x)$ with certainty.

As noted in Section 2, we drop subscript $K$ when $K=\tilde{\mathbb{C}}$ and write FQP instead of $\mathbf{F Q P}{ }_{K}$. Note that $\mathbf{F P} \subseteq \mathbf{F Q P}_{K}$.

We first show a relativized separation result. For a nondeterministic TM $M$ and a string $x$, the notation $\# M(x)$ denotes the number of accepting computation paths of $M$ on input $x$, and let \# TIME $(t)$ be the collection of all functions $\lambda x$.\#M(x) for nondeterministic TMs $M$ running in time at most $t(|x|)$. For a set $T$, set $\# \mathbf{T I M E}(T)=\bigcup_{t \in T} \# \mathbf{T I M E}(t)$.

Theorem 1. There exists a set $A$ such that $\mathbf{F Q P} \mathbf{P}^{A} \nsubseteq \# \mathbf{T I M E}\left(o\left(2^{n}\right)\right)^{A}$. 
Proof. For a set $A$, let $f^{A}(x)=2^{-2|x|} \cdot\left(\left|A \cap \Sigma^{|x|}\right|-\left|\Sigma^{|x|} \backslash A\right|\right)^{2}$ for every $x$. Consider the following oracle QTM $N$. On input $x$ of length $n$, write $0^{n} \circ 0$ in a query tape and apply $H^{n} \oplus I$. Invoke an oracle query and then apply the phase shift $P$ to the oracle answer qubit. Again, make a query to $A$ and apply $H^{n} \oplus I$. Accept $x$ if $N$ observes $\left|0^{n} \circ 0\right\rangle$ in the query tape, and rejects $x$ otherwise. It follows by a simple calculation that $f^{A}(x)=\rho_{N^{A}}(x)$. In particular, $f^{A}$ belongs to $\mathbf{F Q P}{ }^{A}$ if $A$ satisfies the condition that, for every $x$, either $\left|A \cap \Sigma^{|x|}\right|=\left|\Sigma^{|x|} \backslash A\right|$ or $\left|A \cap \Sigma^{|x|}\right| \cdot\left|\Sigma^{|x|} \backslash A\right|=0$.

Let $\left\{M_{i}\right\}_{i \in \mathbb{N}}$ and $\left\{q_{i}\right\}_{i \in \mathbb{N}}$ be two enumerations of all polynomial-time nondeterministic TMs and all nondecreasing functions in $o\left(2^{n}\right)$, respectively, such that each $M_{i}$ halts in time at most $q_{i}(n)$ on all inputs of length $n$. Initially, set $n_{-1}=0$ and $A_{-1}=\varnothing$. At stage $i$ of the construction of $A$, let $n_{i}$ denote the minimal integer satisfying that $n_{i-1}<n_{i}$ and $q_{i}\left(n_{i}\right)<2^{n_{i}-1}$. First let $B=A_{i} \cup \Sigma^{n_{i}-1} 1$. Clearly $f^{B}\left(0^{n_{i}}\right)=1$. If $\# M_{i}^{B}\left(0^{n_{i}}\right) \neq 1$, then define $A_{i}$ to be $B$. Assume otherwise. There exists a unique accepting computation path $p$ of $M_{i}$ on $0^{n_{i}}$. Let $Q$ denote the set of all words that $M_{i}$ queries along path $p$. Since $|Q| \leq q_{i}\left(n_{i}\right)<2^{n_{i}-1}$, there is a subset $C$ of $\Sigma^{n_{i}}$ such that $A_{i-1} \subseteq C$ and $\left|C \cap \Sigma^{n_{i}}\right|=\left|\Sigma^{n_{i}} \backslash C\right|$. For this $C, \# M_{i}^{C}\left(0^{n_{i}}\right) \geq 1$ but $f^{C}\left(0^{n_{i}}\right)=0$.

3.2 Bounded Error Quantum Computable Functions. By replacing exact quantum computation in Definition 1 with bounded-error quantum computation, we can define another quantum function class FBQP.

Definition 2. A function $f$ is in $\mathbf{F B Q P} \mathbf{P}_{K}$ if there exist a constant $\epsilon \in\left(0, \frac{1}{2}\right]$ and a polynomial-time, well-formed $Q T M$ with $K$-amplitudes that, on input $x$, outputs $f(x)$ with probability $\frac{1}{2}+\epsilon$. More generally, for a function $t, \mathbf{F B Q T I M E}(t)$ is defined similarly but by requiring $M$ to halt in time $t(|x|)$. For a set $T$, define $\operatorname{FBQTIME}(T)=\bigcup_{t \in T} \operatorname{FBQTIME}(t)$.

Clearly, $\mathbf{F Q P}_{K} \subseteq \mathbf{F B Q P}_{K}$ and $\mathbf{F B Q P} \mathbf{P}_{\mathbb{Q}} \subseteq \mathbf{F P}^{\text {PSPACE }}$.

Brassard et al.[5] extend Grover's database search algorithm [12] and show how to compute with $\epsilon$-accuracy the amplitude of a given superposition in $O(\sqrt{N})$ time, where $N$ is the size of search space, with high probability. In our terminology, \# TIME $(O(n)) \subseteq$ FBQTIME $\left(O\left(2^{n / 2}\right)\right)$.

Bennett et al.[3], however, show that there exists an NP-set that cannot be recognized by any well-formed QTMs running in time $o\left(2^{n / 2}\right)$ relative to random oracle. This immediately implies that $\# \mathbf{P}^{A} \nsubseteq \mathbf{F B Q T I M E}\left(o\left(2^{n / 2}\right)\right)^{A}$ relative to random oracle $A$.

3.3 Quantum Probability Functions. It is essential in quantum complexity theory to study the behavior of the acceptance probability of a well-formed QTM. Here, we consider quantum functions that output such probabilities. We briefly call these functions quantum probability functions.

Definition 3. A function $f$ from $\Sigma^{*}$ to $[0,1]$ is called a polynomial-time quantum probability function with $K$-amplitudes if there exists a polynomial-time, well-formed $Q T M M$ with $K$-amplitudes such that $f(x)=\rho_{M}(x)$ for all $x$. 
In short, we say that $M$ witnesses in the above definition.

By abusing the existing Valiant's notation \#P , we can coin the new notation $\# \mathbf{Q P}{ }_{K}$ for the class of polynomial-time quantum probability functions.

Definition 4. The notation $\# \mathbf{Q P}_{K}$ denotes the set of all polynomial-time quantum probability functions with $K$-amplitudes.

The lemma below is almost trivial and its proof is left to the reader.

Lemma 1. 1. Every $\{0,1\}$-valued $\mathbf{F Q P} \mathbf{P}_{K}$-function is in $\# \mathbf{Q} \mathbf{P}_{K}$.

2. For every $\# \mathbf{P}$-function $f$, there exist an $\mathbf{F P}$-function $\ell$ and $a \# \mathbf{Q} \mathbf{P}_{\mathbb{Q}}$-function $g$ such that $f(x)=\ell\left(1^{|x|}\right) g(x)$ for every $x$.

3. Let $f \in \# \mathbf{Q P}$ and $s, r \in \mathbf{F Q P}$. Assume that $\operatorname{range}(s) \subseteq(0,1) \cap \mathbb{Q}$ and

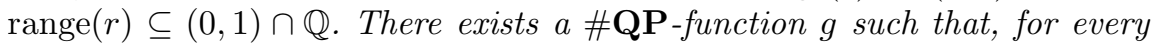
$x, f(x)=s(x)$ iff $g(x)=r(x)$.

Next we show several closure properties of \#QPP-functions.

Lemma 2. Let $f$ and $g$ be any functions in $\# \mathbf{Q P}_{K}$ and $h$ an $\mathbf{F Q P} \mathbf{P}_{K}$-function. (1) If $\alpha, \beta \in K$ satisfy $|\alpha|^{2}+|\beta|^{2}=1$, then $\lambda x$. $\left(|\alpha|^{2} f(x)+|\beta|^{2} g(x)\right)$ is in $\# \mathbf{Q} \mathbf{P}_{K}$. (2) $f \circ h \in \# \mathbf{Q} \mathbf{P}_{K} \cdot$ (3) $f \cdot g$ is in $\# \mathbf{Q} \mathbf{P}_{K}$. (4) If $h$ is polynomially bounded ${ }^{\star \star \star}$, then $\lambda x . f(x)^{h(x)}$ is in $\# \mathbf{Q} \mathbf{P}_{K}$.

Proof Sketch. We prove only the last claim. Let a well-formed $M_{f}$ witness $f$ in time polynomial $q$. Assume that a polynomial $p$ satisfies $h(x) \leq p(|x|)$. On input $x$, run $M_{f} h(x)$ times and idle $q(|x|)$ steps $p(|x|)-h(x)$ times to avoid the timing problem $[4,14]$. Accept $x$ if all the first $h(x)$ runs of $M_{f}$ reach accepting configurations; otherwise, reject $x$.

3.4 Quantum Gap Functions. Notice that \#QP is not closed under subtraction. To compensate the lack of this property, we can introduce the quantum gap functions. A quantum gap function is defined to compute the difference between the acceptance and rejection probabilities of a well-formed QTM.

Definition 5. A function $f$ from $\Sigma^{*}$ to $[-1,1]$ is called a polynomial-time quantum gap function with $K$-amplitudes if there exists a polynomial-time, $K$ amplitude, well-formed QTM $M$ such that, for every $x, f(x)$ is $\|\left|\phi_{x}^{(1)}\right\rangle \|^{2}-$ $\|\left|\phi_{x}^{(0)}\right\rangle \|^{2}$ when $M$ on input $x$ halts in a final superposition $\left|\phi_{x}^{(0)}\right\rangle|0\rangle+\left|\phi_{x}^{(1)}\right\rangle|1\rangle$, where the last qubit represents the content of the output tape of $M$. In other words, $f(x)=2 \rho_{M}(x)-1$.

We use the notation $\mathbf{G a p Q P} \mathbf{P}_{K}$ to denote the collection of such functions.

Definition 6. GapQP ${ }_{K}$ is the set of all polynomial-time quantum gap functions with $K$-amplitudes.

$\star \star \star$ A function from $\Sigma^{*}$ to $\mathbb{N}$ is polynomially bounded if there exists a polynomial $p$ such that $f(x) \leq p(|x|)$ for every $x$. 
For any two sets $\mathcal{F}$ and $\mathcal{G}$ of functions, the notation $\mathcal{F}-\mathcal{G}$ denotes the set of functions of the form $f-g$, where $f \in \mathcal{F}$ and $g \in \mathcal{G}$. The following proposition shows another characterization of $\mathbf{G a p Q P}_{K}$. Recall that $\{0, \pm 1\} \subseteq K \subseteq \mathbb{C}$.

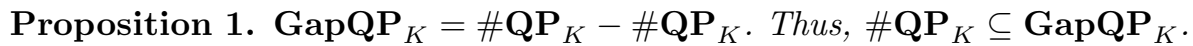

Proof. Clearly, GapQP $\mathbf{P}_{K} \subseteq \# \mathbf{Q P}_{K}-\# \mathbf{Q P}_{K}$. Conversely, assume that there exist two polynomial-time, $K$-amplitude, well-formed QTMs $M_{g}$ and $M_{h}$ satisfying $f(x)=\rho_{M_{g}}(x)-\rho_{M_{h}}(x)$ for all $x$. Consider the following QTM $N$. On input $x, N$ first writes 0 and applies $H$. If $|1\rangle$ is observed, it simulates $M_{g}$. Let $a_{g}$ be its output. Otherwise, $N$ simulates $M_{h}$. Let $a_{h}$ be its output. In the case where $a_{g}=1-a_{h}=1, N$ accepts $x$; otherwise, rejects $x$. The acceptance probability of $N$ is exactly $\frac{1}{2} \rho_{M_{g}}(x)+\frac{1}{2}\left(1-\rho_{M_{h}}(x)\right)$. Thus, the gap $2 \rho_{N}(x)-1$ is exactly $\rho_{M_{g}}(x)-\rho_{M_{h}}(x)$, which equals $f(x)$.

\section{Computational Power of Quantum Gap Functions}

In the previous section, we have introduced quantum gap functions. In this section, we discuss the computational power of these functions.

4.1 Squared Function Theorem. It is shown in [9] that, for every GapPfunction $f$, there exists a polynomial-time, well-formed QTM that accepts input $x$ with probability $2^{-p(|x|)} f(x)^{2}$ for a certain fixed polynomial $p$. This implies that if $f \in \mathbf{G a p P}$ then $\lambda x .2^{-p(|x|)} f(x)^{2} \in$ \#QPP. A slightly different argument demonstrates that if $f \in \mathbf{G a p Q P}$ then $f^{2} \in \# \mathbf{Q P}$. This is a characteristic feature of quantum gap functions.

Theorem 2. (Squared Function Theorem) If $f \in \mathbf{G a p Q P}$ then $f^{2} \in \# \mathbf{Q P}$, where $f^{2}(x)=(f(x))^{2}$ for all $x$.

It is, however, unknown whether GapQP $\cap[0,1]^{\Sigma^{*}} \subseteq \# \mathbf{Q P}$. To show Theorem 2, we utilize the following lemma, whose proof generalizes the argument used in the proof of Proposition 1. See also Lemma 5 in [14].

Lemma 3. (Gap Squaring Lemma) Let $M$ be a well-formed QTM that, on input $x$, halts in time $T(x)$. There exists a well-formed QTM $N$ that, on inputs $x$ given in tape 1 and $1^{T(x)}$ in tape 2 and empty elsewhere, halts in time $O\left(T(x)^{2}\right)$ in a final superposition in which the amplitude of configuration $|x\rangle\left|1^{T(x)}\right\rangle|1\rangle$ is $2 \rho_{M}(x)-1$, where the last qubit is the content of the output tape.

Proof. Let $M$ be the QTM given in the lemma. We define the desired QTM $N$ as follows. First, $N$ simulates $M$ on input $\left(x, 1^{T(x)}\right)$. Assume that $M$ halts in a final superposition $|\phi\rangle=\sum_{y} \alpha_{x, y}|y\rangle\left|b_{y}\right\rangle$, where $y$ ranges all (valid) configurations of $M$ and the qubit $\left|b_{y}\right\rangle$ represents the content of the output tape of $M$. Note that $\rho_{M}(x)=\sum_{y: b_{y}=1}\left|\alpha_{x, y}\right|^{2}$. After $N$ applies the phase shift $P$ to $\left|b_{y}\right\rangle$, we have the superposition $\left|\phi^{\prime}\right\rangle=\sum_{y: b_{y}=1} \alpha_{x, y}|y\rangle|1\rangle-\sum_{y: b_{y}=0} \alpha_{x, y}|y\rangle|0\rangle$. 
There exists a well-formed QTM $M^{R}$ that reverses the computation of $M$ in time $O\left(T(x)^{2}\right)$ on input $\left(x, 1^{T(x)}\right)[14]$. Then, $N$ simulates $M^{R}$ starting with $\left|\phi^{\prime}\right\rangle$. Note that if we apply $M^{R}$ to $|\phi\rangle$ instead, then $M^{R}(|\phi\rangle)=|x\rangle\left|1^{T(x)}\right\rangle|\#\rangle$. Moreover, the inner product of $|\phi\rangle$ and $\left|\phi^{\prime}\right\rangle$ is $\left\langle\phi \mid \phi^{\prime}\right\rangle=\sum_{y: b_{y}=1}\left|\alpha_{x, y}\right|^{2}-\sum_{y: b_{y}=0}\left|\alpha_{x, y}\right|^{2}$, which equals $2 \rho_{M}(x)-1$.

At the end, $N$ outputs 1 (i.e., accept) if it observes exactly the initial configuration; otherwise, $N$ outputs 0 (i.e., reject). Note that the acceptance probability of $N$ is exactly $\left.\langle N(|\phi\rangle)| N\left(\left|\phi^{\prime}\right\rangle\right)\right\rangle$. Since $N$ preserves the inner product, we have $\left.\langle N(|\phi\rangle)| N\left(\left|\phi^{\prime}\right\rangle\right)\right\rangle=\left\langle\phi \mid \phi^{\prime}\right\rangle=2 \rho_{M}(x)-1$.

Now we are ready to prove Theorem 2 .

Proof of Theorem 2. Let $f$ be in GapQP and $M$ a polynomial-time, wellformed QTM such that $f(x)=2 \rho_{M}(x)-1$ for all $x$. It follows from Gap Squaring Lemma that there exists a polynomial-time, well-formed QTM $N$ that halts in a final superposition in which the squared magnitude of the amplitude of $|x\rangle|1\rangle$ is $\left(2 \rho_{M}(x)-1\right)^{2}$, which clearly equals $f^{2}(x)$.

4.2 Approximation of Quantum Gap Functions. Quantum gap functions are closely related to their classical counterpart: gap functions [8]. The following proposition shows the close relationship between GapQP and GapP. Let $\operatorname{sign}(a)$ be 0,1 , and -1 if $a=0, a>0$, and $a<0$, respectively.

Theorem 3. 1. For every $f \in \mathbf{G a p Q P}_{\mathbb{C}}$, there exists a function $g \in \mathbf{G a p P}$ such that, for every $x, f(x)=0$ iff $g(x)=0$.

2. For every $f \in \mathbf{G a p Q P}$ and every polynomial $q$, there exist two functions $k \in \mathbf{G a p P}$ and $\ell \in \mathbf{F P}$ such that $\left|f(x)-\frac{k(x)}{\ell\left(1^{|x|}\right)}\right| \leq 2^{-q(|x|)}$ for all $x$.

3. For every $f \in \mathbf{G a p Q P}_{\mathbb{A} \cap \mathbb{R}}$, there exists a function $g \in \mathbf{G a p P}$ such that $\operatorname{sign}(f(x))=\operatorname{sign}(g(x))$ for all $x$.

4. For every $f \in \mathbf{G a p Q P}_{\mathbb{Q}}$, there exist two functions $k \in \mathbf{G a p P}$ and $\ell \in \mathbf{F P}$ such that $f(x)=\frac{k(x)}{\ell\left(1^{|x|}\right)}$ for all $x$.

To show the theorem, we need the following lemma. For a QTM $M$, let $\operatorname{amp}_{M}(x, C)$ denote the amplitude of configuration $C$ of $M$ on input $x$ in a final superposition. The (complex) conjugate of $M$ is the QTM $M^{*}$ defined exactly as $M$ except that its time-evolution matrix is the complex conjugate of the time-evolution matrix of $M$.

Lemma 4. Let $M$ be a well-formed, synchronous, stationary QTM in normal form with running time $T(x)$ on input $x$. There exists a well-formed $Q T M N$ such that, for every $x,(1) N$ halts in time $O(T(x))$ with one symbol from $\{0,1, ?\}$ in its output tape; (2) $\sum_{C \in D_{x}^{1}} a m p_{N}(x, C)=\rho_{M}(x)$; and (3) $\sum_{C \in D_{x}^{0}} a m p_{N}(x, C)=$ $1-\rho_{M}(x)$, where $D_{x}^{i}$ is the set of all final configurations, of $M$ on $x$, whose output tape consists only of symbol $i \in\{0,1\}$. 
Proof Sketch. The desired QTM $N$ works as follows. On input $x, N$ simulates $M$ on input $x$; when it halts in a final configuration, $N$ starts another round of simulation of $M^{*}$ in a different tape. After $N$ reaches a final configuration, we obtain two final configurations. Then, $N$ (reversibly) checks if both final configurations are identical. If not, $N$ outputs symbol "?" and halts. Assume otherwise. If this unique configuration is an accepting configuration, then $N$ outputs 1 ; otherwise, it outputs 0 .

Proof of Theorem 3. (1) First we note from [15] that, for every $g \in \# \mathbf{Q P}_{\mathbb{C}}$, there exists a $h \in \mathbf{G a p P}$ such that, for every $x, g(x)=0$ iff $h(x)=0$.

Let $f$ be any function in GapQP $\mathbb{C}_{\mathbb{C}}$. By Squared Function Theorem, $f^{2}$ belongs to $\# \mathbf{Q P} \mathbf{P}_{\mathbb{C}}$. Thus, there exists a $h \in \mathbf{G a p P}$ such that, for every $x, f^{2}(x)=0$ iff $h(x)=0$. It immediately follows that $f(x)=0$ iff $h(x)=0$.

(2) Let $f \in$ GapQP. By Lemma 4, it follows that there exists a polynomialtime, well-formed QTM $M$ such that $f(x)$ equals $\sum_{C \in D_{x}^{1}} \operatorname{amp}_{M}(x, C)$ $-\sum_{C \in D_{x}^{0}} a_{m} p_{M}(x, C)$, where $D_{x}^{i}$ is defined in Lemma 4 . Let $r$ be a polynomial that bounds the running time of $M$ and also satisfies $\left|D_{x}^{0} \cup D_{x}^{1}\right| \leq 2^{r(|x|)}$.

Let $x$ be any string of length $n$. Let $\ell(x)=2^{2 r(n)+q(n)+1}$ and $g(x, C)=$ $\operatorname{amp}_{M}(x, C)$. Assume first that there exists a GapQP-function $\tilde{g}$ such that

$\left|g(x, C)-\frac{\tilde{g}(x, C)}{\ell\left(1^{n}\right)}\right| \leq 2^{-r(n)-q(n)-1}$, which implies $\left|\sum_{C \in D_{x}^{i}} g(x, C)-\sum_{C \in D_{x}^{i}} \frac{\tilde{g}(x, C)}{\ell\left(1^{n}\right)}\right|$ $\leq 2^{r(n)} \cdot 2^{-r(n)-q(n)-1}=2^{-q(n)-1}$ for each $i \in\{0,1\}$. The desired GapQPfunction $k$ is defined as $k(x)=\sum_{C \in D_{x}^{1}} \tilde{g}(x, C)-\sum_{C \in D_{x}^{0}} \tilde{g}(x, C)$.

To complete the proof, we show the existence of such $\tilde{g}$. Note that every amplitude of the transition function of $M$ is approximated by a polynomial-time TM. By simulating such a machine in polynomial time, we can get a $2 r(n)+q(n)+1$ bit approximation of the corresponding amplitude to within $2^{-2 r(n)-q(n)-1}$ so that, for every computation path $P$ of $M$ on input $x$, we can compute the approximation $\tilde{\rho}_{P}$ of the amplitude $\rho_{P}$ of path $P$ to within $2^{-r(n)-q(n)-1}$. Let $h(x, P)$ be the integer satisfying $h(x, P)=\ell\left(1^{n}\right)\left|\tilde{\rho}_{P}\right|$. Set $\tilde{g}(x, C)=\sum_{P \in P_{x, C}} h(x, P)$, where $P_{x, C}$ is the collection of all paths of $M$ on $x$ that lead to configuration $C$.

(3) This follows by a modification of the proof of Lemma 6.8 in [1].

(4) In the proof of (2), since $M$ has $\mathbb{Q}$-amplitudes, we can exactly compute the amplitude $\rho_{P}$ of path $P$ and thus, we have $h(x, P)=\ell\left(1^{n}\right)\left|\rho_{P}\right|$. Therefore, $f(x)=\frac{k(x)}{\ell\left(1^{n}\right)}$. See also Theorem 3.1 in [11].

\section{Quantum Complexity Classes}

Using the values of quantum functions, we can introduce a variety of quantum complexity classes. As in the previous sections, when $K=\tilde{\mathbb{C}}$, we drop script $K$.

5.1 GapQP-Definable Complexity Classes. What is the common feature of known quantum complexity classes? Bernstein and Vazirani [4] introduced 
$\mathbf{E Q P}_{K}$ (exact QP) as the collections of sets $S$ such that an $\mathbf{F Q P}{ }_{K}$-function $f$ satisfies $f(x)=S(x)$ for all $x$. Similarly, $\mathbf{B Q P} \mathbf{P}_{K}$ (bounded-error QP) is defined by an $\mathbf{F B Q P} \mathbf{P}_{K}$-function instead of an $\mathbf{F Q P}{ }_{K}$-function. Adleman et al.[1] introduced $\mathbf{N Q P}_{K}$ (nondeterministic QP), which is defined as the collection of sets $S$ such that there exists a \# $\mathbf{Q P} \mathbf{P}_{K}$-function $f$ satisfying that, for every $x, S(x)=1$ iff $f(x)>0$. By a simple observation, $\mathbf{E Q P} \mathbf{P}_{K}, \mathbf{B Q P} \mathbf{P}_{K}$, and $\mathbf{N Q P} \mathbf{P}_{K}$ can be all characterized in terms of GapQP $\mathbf{F}_{K}$-functions.

We introduce a general notion of GapQP-definability in similar spirit that Fenner et al.[8] defined Gap-definability.

Definition 7. A complexity class $\mathcal{C}$ is $\mathrm{GapQP}_{K}$-definable if there exist a pair of disjoint sets $A, R \subseteq \Sigma^{*} \times[0,1]$ such that, for any $S, S \in \mathcal{C}$ iff there exists an $f \in \mathbf{G a p Q P}_{K}$ satisfying that, for every $x$, (i) $x \in S$ implies $(x, f(x)) \in A$ and (ii) $x \notin S$ implies $(x, f(x)) \in R$. We write $\operatorname{Gap} Q P_{K}(A, R)$ to denote this $\mathcal{C}$.

Proposition 2. $\mathbf{E Q P}_{K}, \mathbf{B Q P}_{K}$, and $\mathbf{N Q P}_{K}$ are all GapQP $P_{K}$-definable.

An immediate challenge is to prove or disprove that the following quantum complexity class is GapQP-definable: $\mathbf{W Q P}_{K}$ (wide QP), the collection of sets $A$ such that there exist an $f \in \# \mathbf{Q P}_{K}$ and a $g \in \mathbf{F Q P} \mathbf{P}_{K}$ with range $(g) \subseteq(0,1] \cap \mathbb{Q}$ satisfying $f(x)=A(x) \cdot g(x)$ for every $x$. Notice that we can replace $\# \mathbf{Q P} \mathbf{P}_{K}$ by GapQP $_{K}$. By definition, $\mathbf{E Q P}_{K} \subseteq \mathbf{W Q P}_{K} \subseteq \mathbf{N Q P}_{K}$.

5.2 Sets with Low Information. Let $\mathcal{C}$ be a relativizable complexity class. Consider a set $A$ satisfying $\mathcal{C}^{A}=\mathcal{C}$. Apparently, this set $A$ has low information since it does not help the class $\mathcal{C}$ gain more power. Such a set is called a low set for $\mathcal{C}$. Let low- $\mathcal{C}$ denote the collection of all low sets for $\mathcal{C}$.

Obviously, low-EQP $=\mathbf{E Q P}$. Moreover, since $\mathbf{B Q P} \mathbf{P Q P}^{\mathrm{BQP}}=\mathbf{B Q P}[3]$, we obtain low-BQP $=\mathbf{B Q P}$. However, low-NQP does not appear to coincide with $\mathbf{N Q P}$ since $\mathbf{E Q P} \subseteq$ low- $\mathbf{N Q P} \subseteq \mathbf{N Q P} \cap$ co-NQP. Thus, if $\mathbf{N Q P} \neq$ co-NQP, then low-NQP $\neq \mathbf{N Q P}$.

The following proposition is almost trivial with the fact that $\{0, \pm 1\} \subseteq K$.

Proposition 3. EQP $\mathbf{E}_{K}=$ low-\#QP $\mathbf{P}_{K}=$ low-GapQP ${ }_{K}$.

5.3 Quantum Complexity Classes beyond BQP. We briefly discuss a few quantum complexity classes beyond BQP.

We first note that any BQP set enjoys the amplification property: for every polynomial $p$, there exists a \#QPP-function $f$ such that, for every $x$, if $x \in A$ then $f(x) \geq 1-2^{-p(|x|)}$, and otherwise $0 \leq f(x) \leq 2^{-p(|x|)}$ [3]. Let $\mathbf{A Q P}_{K}$ (amplified QP) be defined similarly by replacing \#QP with $\mathbf{G a p Q P}_{K}$. By definition, $\mathbf{B Q P} \subseteq \mathbf{A Q P}$. Note that if $\mathbf{A Q P} \neq \mathbf{B Q P}$, then $\mathbf{G a p} \mathbf{Q P} \cap[0,1]^{\Sigma^{*}} \neq \# \mathbf{Q P}$. By Proposition 3, AQP $\mathbf{P}_{\mathbb{Q}}$ remains within AWPP, which is defined in [7].

Another natural class beyond $\mathbf{B Q P}$ is $\mathbf{P Q P} \mathbf{P}_{\underline{K}}$ (proper QP) defined as $\operatorname{Gap}_{\operatorname{lop}}(A, \bar{A})$, where $A=\{(x, r) \mid r>0\}$ and $\bar{A}$ is the complement of $A$. Proposition 3, however, yields the coincidence between $\mathbf{P Q P} \mathbf{P}_{\mathbb{Q}}$ and $\mathbf{P P}$.

It is important to note that $\mathbf{A Q P} \mathbf{P}_{\mathbb{C}}$ and $\mathbf{P Q P} \mathbf{P}_{\mathbb{C}}$ are no longer recursive since $\mathbf{B Q P}_{\mathbb{C}}$ is known to be non-recursive [1]. 


\section{Computation with Oracle Queries}

In this section, we study quantum functions that can access oracle sets. In what follows, $r$ denotes an arbitrary function from $\mathbb{N}$ to $\mathbb{N}, R$ a subset of $\mathbb{N}^{\mathbb{N}}$, and $A$ a subset of $\Sigma^{*}$.

6.1 Adaptive and Nonadaptive Queries. We begin with the formal definitions.

Definition 8. A function $f$ is in $\mathbf{F Q} \mathbf{P}^{A[r]}$ if there exists a polynomial-time, well-formed, oracle $Q T M M$ such that, for every $x, M$ on input $x$ computes $f(x)$ using oracle $A$ and makes at most $r(|x|)$ queries on each computation path. The class $\mathbf{F Q P} \mathbf{P}^{A[R]}$ is the union of such sets $\mathbf{F Q P} \mathbf{P}^{A[r]}$ over all $r \in R$.

Note that, when $R=\mathbb{N}^{\mathbb{N}}, \mathbf{F} \mathbf{Q P}{ }^{A[R]}$ coincides with $\mathbf{F Q P} \mathbf{P}^{A}$.

Proposition 4. Let $\mathcal{C}$ be a complexity class that is closed under union, complement, and polynomial-time conjunctive reducibility. If $f \in \# \mathbf{Q} \mathbf{P}^{\mathcal{C}}$ then $\lambda x \cdot \frac{f(x)}{2^{p(|x|)}} \in$ \# $\mathbf{Q P}^{\mathcal{C}}{ }^{[1]}$ for a certain polynomial $p$.

Proof Sketch. Assume that a given QTM $M$ is of "canonical form"; that is, there exists a polynomial $p$ such that $M$ makes $p(n)$ queries of length $p(n)$ on every computation path for $n$ the length of input [14]. Consider the following quantum algorithm. First guess future oracle answers $\left\{a_{i}\right\}_{1 \leq i \leq p(n)}$ and start the simulation of $M$ using $\left\{a_{i}\right\}_{1 \leq i \leq p(n)}$ to answer actual queries $\left\{w_{i}\right\}_{1 \leq i \leq p(n)}$. When $M$ accepts the input, make a single query $\left\langle w_{1}, \cdots, w_{p(n)}, a_{1}, \cdots, a_{p(n)}\right\rangle$ to oracle and verify the correctness of $\left\{a_{i}\right\}_{1 \leq i \leq p(n)}$.

Next, we introduce quantum functions that make nonadaptive (or parallel) queries to oracles. The functions in Definition 8, on the contrary, make adaptive (or sequential) queries.

Definition 9. The class $\mathbf{F Q P} \|_{\|}^{A[r]}$ is the subset of $\mathbf{F Q P} \mathbf{P}^{A[r]}$ with the extra condition that, on each computation path $p$, just before $M$ enters a pre-query state for the first time, it completes a query list ${ }^{\dagger}$ - a list of all query words (separated by a special separator in a distinguished tape) that are possibly ${ }^{\ddagger}$ queried on path p. The notation $\mathbf{F Q P} \mathbf{P}_{\|}^{A[R]}$ denotes the union of $\mathbf{F Q P} \mathbf{P}_{\|}^{A[r]}$ over all $r \in R$.

A similar constraint gives rise to $\mathbf{F B Q P} \mathbf{P}_{\|}^{A[R]}, \# \mathbf{Q} \mathbf{P}_{\|}^{A[R]}$, and $\mathbf{G a p Q} \mathbf{P}_{\|}^{A[R]}$.

The proof of Theorem 1 implies that $\mathbf{F Q P}_{\|}^{A[2]} \not \subset$ \#IME $\left(o\left(2^{n}\right)\right)^{A}$ relative to a certain oracle $A$.

\footnotetext{
$\dagger$ When we say a "query list", we refer to the list completed just before the first query. This query list may be altered afterward to interfere with other computation paths having different query lists.

$\ddagger$ All the words in the query list may not be queried but any word that is queried must be in the query list.
} 
Proposition 5. FQP ${ }^{A[r]} \subseteq \mathbf{F Q P}_{\|}^{A\left[r \cdot 2^{r}\right]}$ for any function $r$ in $O(\log n)$.

Proof. Let $f \in \mathbf{F Q P}^{A[r]}$ and assume that a polynomial-time, well-formed QTM $M$ witnesses $f$ with at most $r(|x|)$ queries to $A$ on each computation path on any input $x$. Consider the following quantum algorithm.

Let $x$ be any input of length $n$. We use a binary string $a$ of length $r(n)$. Initially, we set $a=0^{r(n)}$. In the $k$ th round, $1 \leq k \leq 2^{r(n)}$, we simulate $M$ on input $x$ except that when $M$ makes the $i$ th query, we draw the $i$ th bit of $a$ as its oracle answer. In case where $M$ makes more than $r(n)$ queries, we automatically set their oracle answers to be 0 . We record all query words in an extra tape. After $M$ halts, we increment $a$ lexicographically by one. After $2^{r(n)}$ rounds, we have a query list of size at most $r(n) 2^{r(n)}$. We then simulate $M$ again with using oracle $A$. This last procedure preserves the original quantum interference. Therefore, $f \in \mathbf{F Q P}_{\|}^{A\left[r \cdot 2^{r}\right]}$.

In particular, we have $\mathbf{F Q P} \mathbf{P}^{\mathbf{N Q P}[O(\log n)]} \subseteq \mathbf{F Q P}_{\|}^{\mathbf{N Q P}}$, which is analogous to $\mathbf{F P}^{\mathbf{N P}[O(\log n)] \subseteq \mathbf{F P}_{\|}^{\mathbf{N P}} \text {. It is open, however, whether } \mathbf{F Q P}_{\|}^{A\left[2^{r}\right]} \subseteq \mathbf{F} \mathbf{Q P}} \mathbf{P}^{A[r]}$.

6.2 Separation Result. We show the existence of a set $A$ that separates $\# \mathbf{Q} \mathbf{P}^{A}$ from \# $\mathbf{Q P} \mathbf{P}_{\|}^{A}$. For a string $y$ and a superposition $|\phi\rangle$ of configurations, let $q_{y}(|\phi\rangle)$ denote the query magnitude [3]; that is, the sum of squared magnitudes in $|\phi\rangle$ of configurations which has a pre-query state and query word $y$.

Theorem 4. There exists a set $A$ such that $\# \mathbf{Q P}{ }^{A} \cap\{0,1\}^{\Sigma^{*}} \nsubseteq \# \mathbf{Q P} \mathbf{P}_{\|}^{A}$.

Proof. Define $f^{A}(x)=2^{-|x|} \cdot\left|\left\{y \in \Sigma^{|x|} \mid A\left(x \circ y_{A}\right)=1\right\}\right|$ for $x \in \Sigma^{*}$ and $A \subseteq \Sigma^{*}$, where $y_{A}=A\left(y 0^{|y|}\right) A\left(y 0^{|y|-1} 1\right) A\left(y 0^{|y|-2} 11\right) \cdots A\left(y 1^{|y|}\right)$. We call a set $A$ good at $n$ if, for any pair $y, y^{\prime} \in \Sigma^{n},|y|=\left|y^{\prime}\right|$ implies $y_{A}=y_{A}^{\prime} ; A$ is $\operatorname{good}$ if $A$ is good at every $n$. It follows that, for any good $A, f^{A} \in \# \mathbf{Q P} \mathbf{P}^{A} \cap\{0,1\}^{\Sigma^{*}}$.

We want to construct a good set $A$ such that $f^{A} \notin \# \mathbf{Q P} \mathbf{P}_{\|}^{A}$. Let $\left\{M_{i}\right\}_{i \in \mathbb{N}}$ and $\left\{p_{i}\right\}_{i \in \mathbb{N}}$ be two enumerations of polynomial-time, well-formed QTMs and polynomials such that each $M_{i}$ halts in time $p_{i}(n)$ on all inputs of length $n$. We build by stages a series of disjoint sets $\left\{A_{i}\right\}_{i \in \mathbb{N}}$ and then define $A=\bigcup_{i \in \mathbb{N}} A_{i}$.

For convenience, set $A_{-1}=\varnothing$. Consider stage $i$. Let $n_{i}$ be the minimal integer such that $n_{i-1}<n_{i}$ and $8 p_{i}\left(n_{i}\right)^{4}<2^{n_{i}}$. It suffices to show the existence of a set $A_{i} \subseteq \Sigma^{2 n_{i}} \cup \Sigma^{2 n_{i}+1}$ such that $A_{i}$ is good at $n_{i}$ and $A_{i}\left(0^{n_{i}} \circ y_{A_{i}}\right) \neq \rho_{M_{i}^{A_{i}}}\left(0^{n_{i}}\right)$.

For readability, we omit subscript $i$ in what follows. To draw a contradiction, we assume otherwise. Let $\left|\phi_{j}\right\rangle$ be the superposition of $M$ on input $0^{n}$ at time $j$. For each $y \in \Sigma^{n}$, let $\tilde{q}_{y}$ be the sum of squared magnitudes in any superposition of $M$ 's configurations whose query list contains word $y$. Let $S$ be the set of all $y \in \Sigma^{n}$ such that $M$ on input $0^{n}$ queries $0^{n} y$. In general, $\sum_{y \in S} \tilde{q}_{y} \leq p(n)^{2}$ since the size of each query list is at most $p(n)$. By our assumption, however, $|S|=2^{n}$.

Let $y$ be any string in $S$ and fix $A$ such that $y=y_{A}$. Moreover, let $A_{y}$ be $A$ except that $A_{y}\left(0^{n} y\right)=1-A\left(0^{n} y\right)$. It follows by our assumption that $\rho_{M^{A}}\left(0^{n}\right)=$ $1-\rho_{M^{A_{y}}}\left(0^{n}\right)$. By Theorem 3.3 in [3], since $\left|\rho_{M^{A}}\left(0^{n}\right)-\rho_{M^{A_{y}}}\left(0^{n}\right)\right|=1$, we have 
$\sum_{j=1}^{p(n)-1} q_{y}\left(\left|\phi_{j}\right\rangle\right) \geq \frac{1}{8 p(n)}$. Since $\sum_{j=1}^{p(n)-1} q_{y}\left(\left|\phi_{j}\right\rangle\right) \leq \tilde{q}_{y} \cdot p(n)$, we have $\tilde{q}_{y} \geq \frac{1}{8 p(n)^{2}}$. This immediately draws the conclusion that $|S| \leq 8 p(n)^{4}$, a contradiction.

Acknowledgments: The author would like to thank Andy Yao and Yaoyun Shi for interesting discussion on quantum complexity theory. He is also grateful to anonymous referees for their critical comments on an early draft.

\section{References}

1. L. M. Adleman, J. DeMarrais, and M. A. Huang, Quantum computability, SIAM J. Comput., 26 (1997), 1524-1540.

2. P. Benioff, The computer as a Physical system: A microscopic quantum mechanical Hamiltonian model of computers as represented by Turing machines, J. Stat. Phys., 22 (1980), 563-591.

3. C. H. Bennett, E. Bernstein, G. Brassard, and U. Vazirani, Strengths and weaknesses of quantum computing, SIAM J. Comput., 26 (1997), 1510-1523.

4. E. Bernstein and U. Vazirani, Quantum complexity theory, SIAM J. Comput., 26 (1997), 1411-1473.

5. G. Brassard, P. Høyer, and A. Tapp, Quantum counting, Proc. 25th International Colloquium on Automata, Languages, and Programming, Lecture Notes in Computer Science, Vol.1443, pp.820-831, 1998.

6. D. Deutsch, Quantum theory, the Church-Turing principle, and the universal quantum computer, Proc. Roy. Soc. London, A, 400 (1985), 97-117.

7. S. Fenner, L. Fortnow, S. Kurtz, and L. Li, An oracle builder's toolkit, Proc. 8th IEEE Conference on Structure in Complexity Theory, pp.120-131, 1993.

8. S. Fenner, L. Fortnow, and S. Kurtz, Gap-definable counting classes, J. Comput. and System Sci., 48 (1994), 116-148.

9. S. Fenner, F. Green, S. Homer, and R. Pruim, Determining acceptance possibility for a quantum computation is hard for PH, Proc. 6th Italian Conference on Theoretical Computer Science, World-Scientific, Singapore, pp.241-252, 1998.

10. R. Feynman, Simulating Physics with computers, Intern. J. Theoret. Phys., 21 (1982), 467-488.

11. L. Fortnow and J. Rogers, Complexity limitations on quantum computation, Proc. 13th IEEE Conference on Computational Complexity, pp.202-209, 1998.

12. L. Grover, A fast quantum mechanical algorithm for database search, Proc. 28th ACM Symposium on Theory of Computing, pp.212-219, 1996.

13. L. G. Valiant, The complexity of computing the permanent, Theor. Comput. Sci., 8 (1979), 410-421.

14. T. Yamakami, A foundation of programming a multi-tape quantum Turing machine, Proc. 24th International Symposium on Mathematical Foundations of Computer Science, Lecture Notes in Computer Science, Vol.1672, pp.430-441, 1999. See also LANL quant-ph/9906084.

15. T. Yamakami and A. C. Yao, $\mathrm{NQP}_{\mathbb{C}}=\mathrm{co}-\mathrm{C}=\mathrm{P}$, to appear in Inform. Process. Lett. See also LANL quant-ph/9812032, 1998.

16. A. C. Yao, Quantum circuit complexity, Proc. 34th IEEE Symposium on Foundation of Computer Science, pp.352-361, 1993. 